\title{
Limbic Encephalitis as the First Manifestation of Neurosyphilis: A Diagnostic Challenge
}

\author{
Wadi Bnouhanna, Basma Marzouk, Mounia Rahmani, Maria Benabdeljlil, Saadia Aidi \\ Department of Neurology A and Neuropsychology, Hospital of Specialties, Ibn Sina University Hospital, Faculty of Medicine and \\ Pharmacy of Rabat, Mohammed V University, Rabat, Morocco \\ Email: w.bnouhanna@gmail.com,dr.marzouk.basma@gmail.com,ouniarahmani4@gmail.com,benab.maria@yahoo.fr, \\ aidi_sadia@yahoo.fr
}

How to cite this paper: Bnouhanna, W. Marzouk, B., Rahmani, M., Benabdeljlil, M. and Aidi, S. (2022) Limbic Encephalitis as the First Manifestation of Neurosyphilis: A Diagnostic Challenge. Case Reports in Clinical Medicine, 11, 19-24.

https://doi.org/10.4236/crcm.2022.111004

Received: December 4, 2021

Accepted: January 16, 2022

Published: January 19, 2022

Copyright $\odot 2022$ by author(s) and Scientific Research Publishing Inc. This work is licensed under the Creative Commons Attribution International License (CC BY 4.0).

http://creativecommons.org/licenses/by/4.0/

\begin{abstract}
Limbic encephalitis (LE) is a clinical entity related to a mesial temporal lesion resulting in a combination of anterograde memory dysfunction, behavioral changes, and seizures. The most common causes of limbic encephalitis are Herpes Simplex Virus (HSV) encephalitis, autoimmune encephalitis. Neurosyphilis is an exceptional aetiology. The early diagnosis and management of the disease determine the prognosis. This clinical course highlights the diagnostic challenge limbic encephalitis can cause and the importance of considering neurosyphilis in patients with specific or unspecific neurological symptoms. We report a case of a mesiotemporal form of neurosyphilis revealed by a LE.
\end{abstract}

\section{Keywords}

Limbic Encephalitis, Neurosyphilis, Brain MRI

\section{Introduction}

Limbic encephalitis is a clinical entity related to a mesial temporal lesion resulting in a combination of anterograde memory dysfunction, behavioral changes, and seizures. Cerebral MRI may be normal at an early stage, but the pathognomonic lesions are localized at the limbic region and appear as a hypersignal on T2 and fluid-attenuated inversion recovery (FLAIR) without contrast enhancement. The etiologies of LE are diverse: paraneoplastic, autoimmune, Hashimoto encephalitis, and infectious diseases especially herpetic; however, syphilis is a very rare cause.

\section{Case Report}

A 48-years-old man, with no medical history, presented with epileptic seizures. 
The initial clinical examination found a stiff neck, behavioral disorders, agitation, and temporal-spatial disorientation without sensory-motor impairment. The CT scan with venous angiography was normal. The electroencephalogram (EEG) showed a partial right status epilepticus. The analysis of CSF revealed lymphocytic meningitis (95 white blood cells) with hyperproteinorachia (1.02 g/l) and without hypoglycorachia. Brain MRI showed a T2, FLAIR, and DWI hypersignal at the limbic regions and in the right mesial temporal area, without enhancement after gadolinium injection (Figure 1). Considering the clinico-radiologic findings, two diagnoses were retained: herpetic encephalitis and autoimmune limbic encephalitis. The patient was put under Aciclovir. Due to the negativity of PCR HSV1, 2, on two occasions, and the non-improvement of the neurological state, a pulse of solumedrol $500 \mathrm{mg} /$ day for ten days was added. The finding of anisocoria during his hospitalization prompted us to perform syphilitic serology in the serum with a positive result (TPHA: +, > $2560 \mathrm{IU} / \mathrm{ml}$, VDRL:,$+=1 / 8)$. Syphilitic serology in the cerebrospinal fluid (CSF) was also positive (TPHA +: > 2560; VDRL +: = 1/2) (Table 1). Other paraclinical investigations including hepatitis and HIV serology, anti-thyroid antibodies, and thoraco-abdominopelvic CT scan were normal. The diagnosis of neurosyphilis was made considering clinical, biological, and radiological arguments. The patient was treated with penicillin G 10 MIU/12 hours for 15 days. The evolution was marked by a clear clinical improvement and the normalization of CSF and EEG. The brain MRI control showed a complete radiological cleaning with the occurrence of right hippocampal atrophy with enlargement of the right temporal horn (Figure 2). The follow-up at 3 months showed a positive outcome.

Table 1. Syphilitic serology results in blood and CSF.

\begin{tabular}{ccc}
\hline & Serum & CSF \\
\hline TPHA & $>2560 \mathrm{IU} / \mathrm{ml}$ & $>2560$ \\
VDRL & $1 / 8$ & $1 / 2$ \\
\hline
\end{tabular}

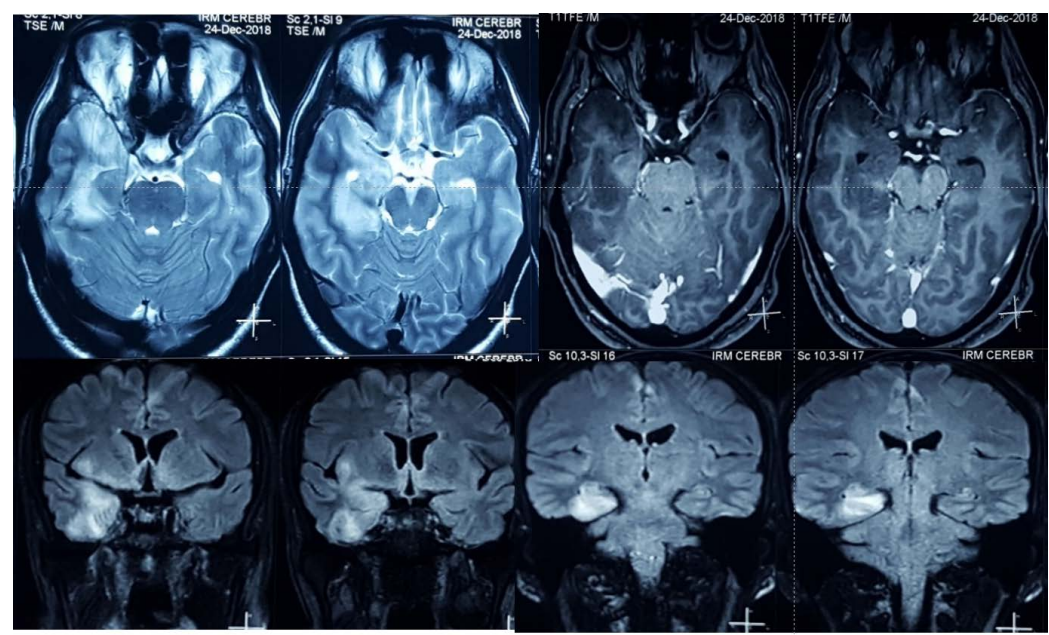

Figure 1. Brain MRI showed a T2, FLAIR, hypersignal at the limbic regions and in the right mesio-temporal area, without enhancement after gadolinum injection. 


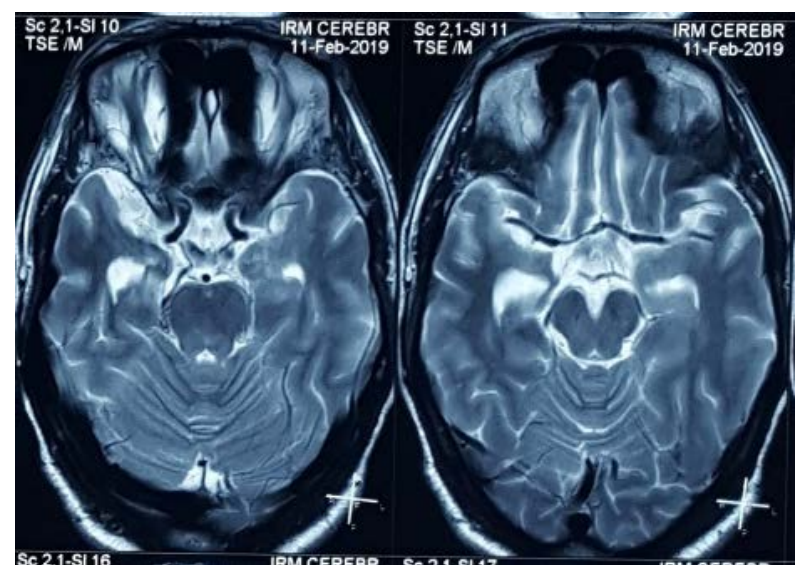

Figure 2. Brain MRI control (axial T2; Coronal FLAIR) showed a complete radiological cleaning with the occurrence of right hippocampal atrophy with enlargement of the right temporal horn.

\section{Discussion}

Our observation suggests 2 points of discussion:

- Etiologic diagnostic challenge in limbic encephalitis.

- Neurosyphilis and limbic encephalitis.

\subsection{Etiologic Diagnostic Challenge in Limbic Encephalitis}

Limbic encephalitis is a syndrome that develops as a rapidly progressive encephalopathy (usually in less than 6 weeks) caused by brain inflammation typically resulting in some combination of [1]:

- Anterograde memory dysfunction and behavioral changes due to involvement of the limbic system.

- Seizure activity arising from one or both temporal lobes (not mandatory).

- MRI signal change within limbic structures, particularly the hippocampus (not mandatory).

The most common causes of limbic encephalitis are herpetic encephalitis and autoimmune limbic encephalitis. Herpetic encephalitis is a brain infection caused by herpes simplex virus type 1 (HVS-1) and is therefore considered a diagnostic and therapeutic emergency. It occurs at any age and presents as an acute temporal necrotizing encephalitis with rapid onset (less than 48 hours) of fever, headache, behavioral changes, language and memory disorders, followed by consciousness disorders [1]. PCR of herpes in the CSF confirms the diagnosis, but it is necessary to know that it can be falsely negative if sampling was done too early (less than 72 hours after the beginning of the symptomatology). Brain MRI shows hypersignal lesions on T2 and FLAIR-affecting limbic and temporo-polar structures +/- hypointense lesions in Susceptibility weighted imaging in these areas. Treatment with intravenous Acyclovir should be started as soon as the diagnosis is evocated. The evolution of herpetic encephalitis is extremely serious: about $20 \%$ mortality with serious neurological sequelae in surviving patients. This is why herpetic encephalitis is the first diagnosis to be made in the 
presence of limbic encephalitis. Other infections including varicella zoster virus (VZV) and rarely mycobacterium tuberculosis can lead to the same clinical picture [2].

Autoimmune Limbic encephalitis (AILE) is an inflammatory disorder of the limbic system. The estimated incidence of encephalitis in high-income countries is about 5 - 10 per 100,000 inhabitants per year [3]. Advances in autoimmune encephalitis (AIE) research have allowed an improvement of the diagnosis, the establishment of curative treatments, and the enhancement of the prognosis. However, the diagnosis of AIE remains difficult because of their varied and nonspecific clinical manifestations. It is therefore important to recognize the suggestive clinical signs that will lead to carrying out additional examinations to make early and adequate therapeutic management. AIE can be triggered by the abnormal expression of an antigen by a tumor or a viral infection, but in most cases, the mechanism of production of autoantibodies is unknown [2]. Several groups of AIE are described according to the nature of antibodies. Some clinical presentations are specific and the severity of symptoms varies depending on the subtypes of encephalitis. It is very important to make the diagnosis because an immunomodulatory treatment initiated early allows partial or complete reversibility of the symptoms [4].

Currently, two major types of autoantibodies have been identified based on whether or not they are associated with cancer [1] [3]:

- "onco-neuronal" antibodies $(\mathrm{Ab})$, with an intracytoplasmic target, are biomarkers of the presence of cancer and associated with paraneoplastic neurological syndromes. They are directed against a generally intracellular antigen expressed ectopically by the tumor cells. Their detections are performed according to standardized techniques that are reproducible and validated on serum.

- Membrane or synaptic target auto-Ab are rarely associated with cancer and frequently associated with limbic encephalitis. Detection techniques are not yet standardized, are carried out in specialized laboratories, and are performed on CSF.

\subsection{Neurosyphilis and Limbic Encephalitis}

Neurosyphilis is a rare condition, less known by young neurologists. Therefore, it remains underdiagnosed due to the unsystematic achievement of TPHA-VDRL serology in patients admitted to the neurological department [5]. Neurosyphilis is a central nervous system (CNS) infection caused by Treponema pallidum invasion and the associated immunological response. Neurosyphilis can occur during any of the two clinical stages [6]:

- Early neurosyphilis occurs within months to a few years after infection, most commonly presenting as meningitis or meningovascular disease.

- Late neurosyphilis occurs years to decades after infection, typically affecting the brain (causing dementia) or the spinal cord (causing tabes dorsalis). 
The diagnosis of neurosyphilis is easy if you think about it: positive syphilitic serology in the CSF. Several clinical signs are usually orienting: pupillary anomalies, the most typical of which is the Argyll-Robertson sign, as in our patient, proprioceptive disorders of the lower limbs, chin tremor and cognitive disorders.

Brain MRI findings for neurosyphilis are non-specific, and no radiologic or pathognomonic findings have been reported. More common neuroimaging findings for neurosyphilis are brain atrophy, cerebral infarctions, leptomeningeal enhancement, or non-specific white matter lesions. Occasionally it can occur by a hypersignal T2/FLAIR on the mesial temporal lobe which raises a problem of differential diagnosis with herpes simplex or limbic encephalitis [6] [7].

Based on a literature search in the PubMed database using the keywords "neurosyphilis" and "limbic encephalitis", only 13 case reports were found. The authors emphasize the difficulty of diagnosis caused by a diagnostic delay. Neurosyphilis is also known as the "great imitator" this is why we believe that the syphilis serology must be realized in all patients with an encephalitis syndrome, in particular, those with a limbic attack since it will first allow an early diagnosis and management and secondly prevent neurological sequelae [8] [9] [10].

Our clinical observation is particularly interesting for two reasons: the clinical presentation associating clinical features of limbic encephalitis with subclinical status epilepticus and the demonstrated therapeutic response based on clinical, biological, and radiological arguments.

\section{Conclusion}

In conclusion, neurologists must recognize syphilis as a "great imitator". Thus, it is vital to retain neurosyphilis as a differential diagnosis in patients with limbic encephalitis and a mesio-temporal lobe hypersignal to provide early diagnosis and treatment, and a better prognosis.

\section{Acknowledgements}

We thank our colleagues from the Neurology A and Neuropsychology department as well as the Neuroradiology team of the Hospital of Specialties, Ibn Sina University Hospital for their and expertise that greatly assisted our research.

\section{Conflicts of Interest}

The authors declare no conflicts of interest regarding the publication of this paper.

\section{References}

[1] Venkatesan, A., Michael, B.D., Probasco, J.C., Geocadin, R. and Solomon, T. (2019) Acute Encephalitis in Immunocompetent Adults. The Lancet, 393, 702-716. https://doi.org/10.1016/S0140-6736(18)32526-1

[2] Derouich, I., Messouak, O. and Belahsen, M.F. (2013) Syphilitic Limbic Encephalitis Revealed by Status Epilepticus. BMJ Case Reports, 2013, bcr2012008073.

https://doi.org/10.1136/bcr-2012-008073 
[3] Graus, F., Titulaer, M.J., Balu, R., Benseler, S., Bien, C.G., Cellucci, T., et al. (2016) A Clinical Approach to Diagnosis of Autoimmune Encephalitis. The Lancet Neurology, 15, 391-404. https://doi.org/10.1016/S1474-4422(15)00401-9

[4] Beiruti, K., Abu Awad, A., Keigler, G., Ryder, C.H. and Shahien, R. (2019) Atypical Development of Neurosyphilis Mimicking Limbic Encephalitis. International Journal of STD \& AIDS, 30, 194-197. https://doi.org/10.1177/0956462418797873

[5] Tiwana, H. and Ahmed, A. (2018) Neurosyphilis: Mighty Imitator Forays with Benign Presentation and Unique Neuroimaging Findings. Sex Health, 15, 358-360. https://doi.org/10.1071/SH17088

[6] Mandetta Pettengill, A.L., Littig, I., Padilha, I.G., Padula, M., Torres Pacheco, F., Nunes, R.H., Amaral, L., Maia Junior, A.C.M. and Rocha, A.J. (2018) Neurosyphilis in MRI: A Pictorial Review to Remember the Old Faces of the Great Mimicker. ECR 2018/C-1319, Sao Paulo.

[7] Jeong, Y.M., Hwang, H.Y. and Kim, H.S. (2009) MRI of Neurosyphilis Presenting as Mesiotemporal Abnormalities: A Case Report. Korean Journal of Radiology, 10, 310-312. https://doi.org/10.3348/kjr.2009.10.3.310

[8] Gaud, S., Sauvée, M., Muresan, M., Gospodaru, N., Foscolo, S. and Debouverie, M. (2011) Left Mesiotemporal Lesions and Anterograde Memory Impairement: A Case of Neurosyphilis. Revue Neurologique, 167, 833-836. https://doi.org/10.1016/j.neurol.2011.03.006

[9] Geisler, F., et al. (2012) Auto-Antibody-Negative Limbic-Like Encephalitis as the First Manifestation of Neurosyphilis. Clinical Neurology and Neurosurgery, 115, 1485-1487. https://doi.org/10.1016/j.clineuro.2012.11.012

[10] Chahine, L.M., Khoriaty, R.N., Tomford, W.J. and Hussain, M.S. (2011) The Changing Face of Neurosyphilis. International Journal of Stroke, 6, 136-143. https://doi.org/10.1111/j.1747-4949.2010.00568.x 